\title{
Bedside adherence to clinical practice guidelines for enteral nutrition in critically ill patients receiving mechanical ventilation: a prospective, multi-centre, observational study
}

Jean-Pierre Quenot ${ }^{*}$, Gaetan Plantefeve ${ }^{2}$, Jean-Luc Baudel ${ }^{3}$, Isabelle Camilatto ${ }^{4}$, Emmanuelle Bertholet ${ }^{5}$, Romain Cailliod ${ }^{6}$, Jean Reignier ${ }^{7}$, Jean-Philippe Rigaud ${ }^{8}$

\begin{abstract}
Introduction: The primary aim was to measure the amount of nutrients required, prescribed and actually administered in critically ill patients. Secondary aims were to assess adherence to clinical practice guidelines, and investigate factors leading to non-adherence.

Methods: Observational, multicenter, prospective study, including 203 patients in a total of 19 intensive care units in France. The prescribed calorie supply was compared with the theoretical minimal required calorie intake ( $25 \mathrm{Kcal} / \mathrm{Kg} /$ day) and with the supply actually delivered to the patient to calculate the ratio of calories prescribed/ required and the ratio of calories delivered/prescribed. Clinical factors suspected to influence enteral nutrition were analyzed by univariate and multivariate analysis.

Results: The median ratio of prescribed/required calories per day was 43 [37-54] at day 1 and increased until day 7. From day 4 until the end of the study, the median ratio was $>80 \%$. The median ratio of delivered/prescribed per day was $>80 \%$ for all 7 days from the start of enteral nutrition. Among the variables tested (hospital type, use of a local nutrition protocol, sedation, vasoactive drugs, number of interruptions of enteral nutrition and measurement of gastric residual volume), only measurement of residual volume was significant by univariate analysis. This was confirmed by multivariate analysis, where gastric residual volume measurement was the only variable independently associated with the ratio of delivered/prescribed calories $(\mathrm{OR}=1.38 ; 95 \% \mathrm{Cl}, 1.12-2.10$, $\mathrm{p}=.024)$.

Conclusions: The translation of clinical research and recommendations for enteral nutrition into routine bedside practice in critically ill patients receiving mechanical ventilation was satisfactory, but could probably be improved with a multidisciplinary approach.
\end{abstract}

\section{Introduction}

Nutritional support is now considered as a standard of care for intensive care unit (ICU) patients and has been the first-line choice for more than two decades [1]. The generally accepted goals of nutritional delivery in critically ill patients are to provide nutritional therapy consistent with the patient's condition, prevent nutrient deficiencies, avoid complications related to nutrition

\footnotetext{
* Correspondence: jean-pierre.quenot@chu-dijon.fr
'Service de Réanimation Médicale, Bocage University Hospital, Boulevard de

* Correspondence: jean-pierre.quenot@chu-dijon.fr
'Service de Réanimation Médicale, Bocage University Hospital, Boulevard de Lattre de Tassigny, 21079 Dijon, France
}

(c) 2010 Quenot et al.; licensee BioMed Central Ltd. This is an open access article distributed under the terms of the Creative Commons Attribution License (http://creativecommons.org/licenses/by/2.0), which permits unrestricted use, distribution, and reproduction in any medium, provided the original work is properly cited. sive care doctors aim to administer $25 \mathrm{Kcal} / \mathrm{Kg} /$ day, an energy target in line with recent recommendations [1-3]. Unfortunately, a number of factors render the provision of optimal enteral nutrition difficult, such as insufficient caloric targets, gastrointestinal dysfunction such as vomiting and diarrhea, repeated procedures and surgeries associated with interruption of enteral nutrition, feeding tube displacement, inadequate routine nursing procedures with delayed administration of the enteral feed, or premature enteral nutrition withdrawal [4-6]. 
The implementation of feeding protocols has been proposed as a strategy to optimize adequate delivery of nutritional support $[7,8]$. Despite a number of corrective measures proposed in recent years, exclusive enteral nutrition in ICU patients remains associated with nutritional deficiencies, and is correlated with impaired short- and long-term clinical outcomes $[9,10]$. To assess the translation of recommendations $[1-3,7,8]$ into routine critical care, we measured the amount of nutrients required, prescribed and actually delivered in critically ill patients. Furthermore, we sought to identify the reasons for discrepancies between prescriptions and requirements, and between prescriptions and actual delivery of nutrition, through a prospective, observational, multicenter study. Preliminary results were presented at the $37^{\text {th }}$ Congress of the Société de Réanimation de langue Française (SRLF, French-speaking Society of Intensive Care) in Paris, in January 2009.

\section{Materials and methods Study design}

An observational, prospective, multicentre study was conducted in 19 ICUs in France (see acknowledgements for complete list of participating centers). In early 2008, the Clinical and Epidemiology Research Commission (CERC) of the French-speaking Society for Intensive Care (SRLF) posted on its website a call for nurses to participate in a working group to evaluate practices in enteral nutrition and adherence to national guidelines published by the SRLF $[1-3,7,8]$. All 44 respondents, representing 24 French ICUs, were included in the working group, which also included four critical care physicians (members of the CERC). The study protocol (study variables, inclusion and exclusion criteria etc) was developed with the working group during a one-day meeting. No specific protocol for enteral nutrition was stipulated, in order to preserve the 'real world' nature of the observations. The members of the working group constituted the participating centers. As this observational study required no deviation from routine medical practice, institutional review board approval was not required. The study was approved by the Ethics Committee of the SRLF.

\section{Patient population}

Over a period of two months (15 August to 15 October 2008) consecutive patients receiving mechanical ventilation and without contraindication to initiation of enteral nutrition (e.g., gastrointestinal bleeding, ileus, suspected perforation, abdominal surgery, prone positioning) or to insertion of a small-bore feeding tube (e.g. active variceal bleeding) were considered eligible for the study. Patients receiving non-invasive mechanical ventilation or parenteral nutrition were excluded. Decisions related to care, time of insertion, type of feeding tube, type of enteral formula, and use of prokinetic medication were guided by the multidisciplinary team caring for the patient. All patients received enteral nutrition via continuous infusion by a feeding pump. The amount of enteral nutrition delivered was quantified daily. Daily caloric intake was determined by multiplying the total amount of enteral nutrition delivered by the caloric content of the formula(s) and was recorded every morning.

A local protocol for enteral nutrition (no details available) previously existed and was applied in 12 ICUs, while only seven ICUs systematically measured gastric residual volume (GRV).

\section{Data collection}

For each patient, the following data were recorded on admission: age, gender, body mass index $(B M I=$ weight in $\mathrm{Kg}$ divided by height in meters squared), primary diagnosis and Simplified Acute Physiology Score (SAPS) II [11]. Prescriptions of sedation and vasoactive drugs were also recorded. The reasons for interruptions of enteral nutrition were recorded (weaning, radiology, emesis, diarrhea, problems with the small-bore feeding tube etc) for the seven days of the study period.

The duration of mechanical ventilation was also recorded.

Each day until day seven (or until patients were extubated, whichever came first), the amount of nutrients prescribed enterally and the amount of nutrients actually delivered to each patient was recorded by the nurses in each ICU. The optimal minimal calorie supply was set at $25 \mathrm{Kcal} / \mathrm{Kg} /$ day in accordance with current guidelines [1-3]. For obese patients (BMI $>30 \mathrm{Kg} / \mathrm{m}^{2}$ ), optimal calorie intake was calculated for a theoretical weight corresponding to a BMI of $30 \mathrm{~kg} / \mathrm{m}^{2}$. The Harris-Benedict equation adjusted for stress factors was not used in participating ICUs for calculation of required calories. Length of stay in the ICU and in-hospital, as well as mortality were also recorded.

\section{End points for enteral nutrition efficacy}

The primary objective of this study was to calculate the ratio of prescribed to required calories, and the ratio of calories actually delivered to calories prescribed. The prescribed calorie supply was compared with the theoretical minimum required calorie intake $(25 \mathrm{Kcal} / \mathrm{Kg} /$ day), and the calorie supply actually administered to the patient was compared with the prescribed amount. As a secondary endpoint, we analyzed factors likely to influence enteral nutrition and contribute to non-adherence to published guidelines for enteral nutrition.

\section{Data evaluation and quality control}

All data except SAPS II and patient outcome were collected by the investigating nurses in each ICU. An 
independent research assistant entered data into a database using a specific computer program (Microsoft Excel, Microsoft Corp., Redmond, WA, USA). The program included reliability checks based on ranges for all data, and logical checks for inconsistencies and missing data. The members of the CERC carried out extensive data cleaning, and queries were addressed to the investigators for questionable or missing data.

\section{Statistical analysis}

Continuous variables are reported as mean \pm standard deviation or median (interquartile range). The median ratios of prescribed/required calories and delivered/prescribed calories were determined for the first seven days after the start of enteral nutrition or until the patient was extubated (whichever occurred first).

Clinical factors suspected to influence enteral nutrition (hospital type, use of a local nutrition protocol, sedation, vasoactive drugs, measured gastric residual volume and number of interruptions (divided into two classes $<5$ and $>5$ )) were analyzed using the MannWhitney $U$ test. Clinical factors suspected to influence the ratio of calories delivered/prescribed were analyzed by multivariate logistic regression. Variables associated with the ratio of delivered/prescribed calories by univariate analysis $(P<0.10)$ were entered into a stepwise logistic regression.

A $P$ value less than 0.05 was considered significant. Statistical analyses were performed using SAS v 8.2 software (SAS Institute, Cary, NC, USA).

\section{Results}

\section{Characteristics of study population}

A total of 203 patients were included in the study (Table 1). Mean age was $62 \pm 18$ years; 134 (66\%) were men. Mean SAPS II score on ICU admission was $53 \pm$ 18 points. Mean BMI was $27 \pm 8 \mathrm{Kg} / \mathrm{m}^{2}$. The participating ICUs comprised university and/or regional hospitals ( $\mathrm{n}=10,52 \%)$, and general (non academic) hospitals $(\mathrm{n}=9,48 \%)$. There were $6(31 \%)$ mixed medico-surgical and 13 (69\%) medical ICUs. The mean number of beds in ICUs was $14 \pm 3$.

\section{Primary endpoint: calories prescribed, required and actually delivered}

The median ratio of prescribed/required calories per day was 43 (37 to 54) on day one and increased until day seven (Table 2). From day four until the end of the study, the median ratio was more than $80 \%$. The analysis concerned all 203 patients on day one, and decreased to 110 patients on day seven, due to interruptions to enteral nutrition and/or extubation in some patients.
Table 1 Patient characteristics

\begin{tabular}{|c|c|}
\hline Number of patients & 203 \\
\hline \multicolumn{2}{|l|}{ Hospital type (n) } \\
\hline Academic & 89 \\
\hline Community & 114 \\
\hline Age (years) & $62 \pm 18$ \\
\hline Gender (male/female) & $134 / 69$ \\
\hline SAPS \| (points) & $53 \pm 18$ \\
\hline Body mass index $\left(\mathrm{kg} / \mathrm{m}^{2}\right)$ & $27 \pm 8$ \\
\hline \multicolumn{2}{|l|}{ Primary diagnosis, n (\%) } \\
\hline Respiratory & $65(32)$ \\
\hline Cardiovascular & $11(5)$ \\
\hline Neurologic & $54(27)$ \\
\hline Renal & $4(2)$ \\
\hline Post surgical & $7(3)$ \\
\hline Septic shock & $28(14)$ \\
\hline Traumatologic & $10(5)$ \\
\hline Burns & $1(0.5)$ \\
\hline Other & $23(11)$ \\
\hline Mechanical ventilation (days) & $12 \pm 9$ \\
\hline Length of ICU stay (days) & $15 \pm 13$ \\
\hline Length of hospital stay (days) & $28 \pm 19$ \\
\hline ICU mortality, n (\%) & $50(25)$ \\
\hline In-hospital mortality, n (\%) & $65(32)$ \\
\hline
\end{tabular}

Continuous variables are reported as mean \pm standard deviation and categorical variables as number of patients (percent)

ICU: intensive care unit; SAPS II, simplified acute physiologic score II.

The median ratio of delivered/prescribed per day was more than $80 \%$ over the seven days from the start of enteral nutrition.

\section{Secondary endpoint: factors suspected to influence enteral nutrition}

We evaluated by univariate analysis the following variables, considered likely to influence enteral nutrition, and contribute to non-adherence to feeding guidelines: hospital type, use of a local nutrition protocol, sedation, vasoactive drugs, number of interruptions, and measurement of GRV (Table 3). Among the variables tested, only the systematic measurement of GRV was significantly associated by univariate analysis with the mean ratio of prescribed/required and delivered/prescribed calories: when GRV was not measured, there was a significantly higher mean ratio of prescribed/required and delivered/prescribed calories $(P<0.05)$. This was confirmed by multivariate analysis, where GRV measurement was the only variable independently associated with the ratio of delivered/prescribed calories (odd ratio $=1.38 ; 95 \%$ confidence interval $=1.12$ to 2.10 , $P=0.024)$. In practice, when GRV is measured, there is a $38 \%$ increase in the risk of having a low ratio of delivered/prescribed calories. 
Table 2 Ratio of prescribed to required calories, ratio of delivered to prescribed calories and ratio of delivered to required calories per day

\begin{tabular}{lcccc}
\hline Day & Number of patients on each day & \% of prescribed/required & \% of delivered/prescribed & \% of delivered/required \\
\hline 1 & 203 & $43(37-54)$ & $85(77-92)$ & $36(29-44)$ \\
2 & 189 & $67(59-76)$ & $85(76-91)$ & $57(48-65)$ \\
3 & 166 & $73(65-88)$ & $90(80-98)$ & $66(58-73)$ \\
4 & 148 & $80(71-91)$ & $90(79-97)$ & $72(65-80)$ \\
5 & 130 & $86(78-93)$ & $93(82-101)$ & $80(71-88)$ \\
6 & 116 & $88(79-95)$ & $90(79-99)$ & $79(70-86)$ \\
7 & 110 & $87(78-94)$ & $93(81-102)$ & $81(73-88)$ \\
\hline
\end{tabular}

Values are expressed as median (interquartile range).

\section{Discussion}

This is the first multicenter study to assess the level of bedside adherence to clinical practice guidelines for enteral nutrition in critically ill patients receiving mechanical ventilation further to the publication of recent guidelines [1-3].

The main finding of our study is a good ratio of calories actually delivered/prescribed $(>80 \%)$ and calories prescribed/required ( $>80 \%$ ), notably after 72 hours. These results are better than those observed in recent studies in similar populations $[5-8,12,13]$. We observed a satisfactory ratio of delivered/prescribed calories, exceeding $80 \%$, indicating that in general, medical prescriptions are accurately applied by the ICU team over the first seven days.

The main objective of nutrition in critical care is to obtain a calorie content of 25 to $35 \mathrm{Kcal} / \mathrm{Kg} /$ day at two to three days [1-3]. The amount of calories is based on measurement of oxygen consumption (indirect calorimetry) as the reference method, but this requires costly equipment and technical skills that are not widely available, as well as being time-consuming [14]. Another method is the assessment of resting energy expenditure using the Harris-Benedict formula [15], which is a simple formula that takes into account the patient's weight, height, age, and gender.

Previous reports have shown that the calorie supply prescribed and that actually delivered are often below the patients' theoretical needs, because of late initiation, unjustified or excessively long interruptions, diagnostic procedures, airway management, mechanical problems, and failure to reinstill GRV samples $[5,16,17]$. The tolerability of enteral nutrition is sometimes poor, especially in case of treatment with vasoactive drugs, shock, or sedation, or in case of vomiting, repeated interruption of enteral feeding, or upper digestive intolerance $[13,17,18]$.

Table 3 Variables influencing the total ratio of delivered to prescribed calories over the seven-day study period by univariate analysis

\begin{tabular}{|c|c|c|c|c|c|}
\hline Variable & Number of patients & $\%$ prescribed/required & $P$ value & $\%$ delivered/prescribed & $P$ value \\
\hline Hospital type & & & 0.91 & & 0.67 \\
\hline Academic & 89 & 70 (59-78) & & $86(79-97)$ & \\
\hline Community & 114 & $72(63-80)$ & & $87(80-97)$ & \\
\hline Local protocol & & & 0.38 & & 0.94 \\
\hline Yes & 137 & $73(65-79)$ & & $88(81-100)$ & \\
\hline No & 66 & $66(59-73)$ & & 84 (79-98) & \\
\hline Sedation & & & 0.86 & & 0.03 \\
\hline Yes & 150 & $66(58-78)$ & & $89(82-101)$ & \\
\hline No & 53 & $62(54-71)$ & & $80(71-87)$ & \\
\hline Vasoactive drugs & & & 0.32 & & 0.77 \\
\hline Yes & 102 & $70(59-79)$ & & 88 (81-99) & \\
\hline No & 101 & $72(61-80)$ & & $86(79-92)$ & \\
\hline GRV measured & & & 0.002 & & 0.01 \\
\hline Yes & 135 & $68(59-77)$ & & $83(76-89)$ & \\
\hline No & 68 & $77(69-84)$ & & $95(90-104)$ & \\
\hline \multicolumn{6}{|c|}{ Number of interruptions } \\
\hline$<5$ & 180 & $71(63-79)$ & 0.42 & $71(66-78)$ & 0.08 \\
\hline$>5$ & 23 & $69(58-75)$ & & $65(59-72)$ & \\
\hline
\end{tabular}

Values are expressed as median (interquartile range); GRV: gastric residual volume 
In our study, the only factor that significantly influenced the prescribed calories and the level of actually delivered calories by univariate analysis was the measurement of GRV. This could be explained by the fact that GRV measurement by ICU nurses is either systematic (i.e. stipulated by local protocol), particularly at the time of initiation of enteral nutrition; or else applied in case of regurgitation, which hinders the achievement of daily calorie intake goals. In this case, the nurses tend to lower the flow rate, or even stop enteral nutrition altogether.

In one recent report, immediate introduction of the optimal dose of enteral nutrition was associated with significantly more episodes of GRV of more than $300 \mathrm{ml}$ and with a trend towards more frequent use of prokinetic agents [19]. The impact of GRV on the risk of serious adverse events is controversial, and controversy persists regarding the threshold predictive of unfavourable outcome (about $250 \mathrm{ml}$ ) [20]. A recent study has shown a non-consistent relation between aspiration and GRVs [21]. The role of gastrointestinal dysfunction might have been reduced by the fact that the decision to start, increase, reduce, or stop enteral nutrition was made by the physician according to the patient's clinical condition, especially the gastrointestinal tract status (vomiting, diarrhea, or abdominal pain or distension). Our study was not designed to evaluate gastrointestinal tolerance to enteral feeding, because such an evaluation would have required a standardized protocol for enteral nutrition to be applied in all participating centers.

Interestingly, we observed a significantly higher ratio of delivered/prescribed calories in sedated patients. This could be explained by the fact that physicians tend to prescribe less enteral nutrition because of the risk of regurgitation among these patients, and thus, ICU nurses would generally have proceeded as usual in accordance with their standard practice or as stipulated in any local protocol.

Recent evidence suggests that even with the best intensive educational programs to increase compliance with enteral nutrition guidelines, patients receive only $50 \%$ of the prescribed requirements [22].

In our study, the existence of a local protocol had no effect on the total percentage of calories delivered or prescribed, perhaps because published guidelines are simple and easily applicable [1-3]. Clinical trials to assess interventions and outcomes in enteral nutrition may not be applicable to everyday practice, given that delivery of prescribed enteral nutrition is commonly incomplete. Therefore, we believe that the results of this 'real world' study are a powerful tool to inform about the processes used to feed patients [23].

Most procedure and radiological studies require the patient to be supine, a requirement that interrupts enteral nutrition because of the increased risk of aspiration. Together, procedures and radiological studies accounted for $13 \%$ of the interruptions in enteral nutrition [13].

We observed in our study a discrepancy between required and prescribed calories, which can most probably be explained by under prescription on the part of the physicians. Insufficient information, notably absence of BMI data at admission, likely led to sub-optimal prescription.

In our study, we did not assess the effect of enteral nutrition on patient outcome. Few studies have demonstrated the capacity of enteral nutrition to reduce infectious complications, improve nutritional endpoints, or decrease mortality $[1,23]$. A recent study [4] demonstrated that although successful implementation of the guidelines resulted in a significant change in practice, it did not lead to reduced hospital mortality in critically ill patients.

\section{Study limitations}

There are several limitations associated with the methods used in this study. The protocol used in participating ICUs was not stipulated in detail, notably as regards use of the Harris-Benedict formula [15], prokinetic medication or measurement of GRV. Also, local protocols were generally based on the same French and international recommendations [1-3]. The results would likely have been significantly different if a reference level for theoretical calorie requirements above $25 \mathrm{Kcal} / \mathrm{kg}$ /day had been used. It should be noted that there was a considerable reduction (about 50\%) in the number of participants after day three, which undoubtedly reduces the power of this study and the results should be interpreted with care. Also, it should be noted that we were unable to calculate the caloric uptake contained in infusions or the lipid content of propofol infusions.

Furthermore, the patient population was predominantly non-surgical, and any conclusions are restricted to this population and the results of this study cannot be extrapolated to other patient types or all other ICUs in France, because the patient populations may be significantly different in other centers.

Finally, although multivariate analysis was performed, its results should be interpreted with caution, because this was an observational study, and it is impossible to take into account all confounding factors.

\section{Conclusions}

This study is in line with efforts at European level to evaluate professional practices, and quantify the differences between what is recommended in clinical guidelines and/or the medical literature, and what actually happens in daily routine practice at the bedside. 
We proposed a multidisciplinary approach to nutritional support including nurses, dieticians, and pharmacists, with regular training of medical staff involved in nutrition support prescription and delivery. A comprehensive review of routine practice in ICUs might help to achieve optimal nutrition care for critically ill patients.

The translation of clinical research and recommendations for enteral nutrition into routine critical care at the bedside in critically ill patients receiving mechanical ventilation was satisfactory, but could likely be improved with the use of a multidisciplinary approach.

\section{Key messages}

- In patients receiving enteral nutrition, the calorie supply prescribed and that actually delivered are often below the patients' theoretical needs.

- We performed an observational, multicenter study in a representative sample of ICUs to evaluate theoretical calorie requirements, calories prescribed, and actual calories delivered in ICU patients, in light of guidelines for enteral nutrition.

- We observed a good ratio of calories actually delivered/prescribed $(>80 \%)$ and calories prescribed/ required $(>80 \%)$, notably after 72 hours, indicating that in general, medical prescriptions are accurately applied by the ICU team over the first seven days. - In our study, the only factor that significantly influenced the prescribed calories and the level of actually delivered calories by univariate analysis was the measurement of GRV. This was confirmed by multivariate analysis, where GRV measurement was the only variable independently associated with the ratio of delivered/prescribed calories.

\section{Abbreviations}

BMI: body mass index; CERC: Clinical and Epidemiology Research Commission; GRV: gastric residual volume; ICU: intensive care unit; SAPS: Simplified Acute Physiology Score; SRLF: Société de Réanimation de langue Française.

\section{Acknowledgements}

All authors are members of the CERC-SRLF study group.

We thank the Société de Réanimation de Langue Française, Chantal Sevens and Florence Neels for meeting organisation. We thank Agnes Clivet for the data base. We gratefully thank all the participating members of the study (see appendix). We thank Fiona Ecarnot for translation and editorial assistance.

All authors except RC participated in the elaboration of the study protocol and the collection of data. RC performed the analysis of data. All authors revised the manuscript for critical content and approved the final version. Members of the Epidemiology and Clinical Research Commission (Commission d'Epidémiologie et de Recherche Clinique, CERC) of the SRLF were: JP. Rigaud (Dieppe, France), G. Plantefeve (Argenteuil, France), JL Baudel (Paris-Saint-Antoine, France), I. Camilatto (Mulhouse, France), E. Bertholet (Lyon, France), F. Bruneel (Versailles, France), B. Lambermont, (Sart Tilman, Belgique), L. Liaudet (Lausanne, Suisse), JM. Liet (Nantes, France), C. Vinsonneau (Paris-Cochin), J. Reignier, (La Roche/Yon, France).

Investigators (Listed Alphabetically)
Argenteuil (J. Salor), Belfort (G. Zagar, N. Himer, M. Germain), Bobigny (J. Courtois, C. Vallon), Boulogne (A. Hnyluck), Colombes (L. Bloch), Dieppe (E. Duputel, S. Ple), Dijon (M. Ogeas, C. Milard), Garches (N. Lemaire), La Roche sur Yon (Y. Alcourt), Lyon (P. Sarre, E. Bertholet), Marseille (K. Piriou), Montpellier (A. Prades), Mulhouse (E. Camilatto, S. Cubizolle, J. Dangel), Nancy (E. Gaujard, M. Lesny), Nantes (S. Boedec, H. Martineau), Orléans (C. Loiseau, V. Mazelaygue, L. Boureau, V. Noel, A. Languille), Paris-St Antoine (V. Soulie, P. Fiori, C. Bevort), Paris-Lariboisière (S. Kerever), Cochin (A. Ben Amara), Poitiers (D. Coindre, S. Leboursicot), Rouen (L. Douville, C. Gricourt, M. Paradis, AL. Thuret), Strasbourg (S. L'Hotelier), Toulon (V. Bosca), Versailles (l. Rosello)

\section{Author details}

'Service de Réanimation Médicale, Bocage University Hospital, Boulevard de Lattre de Tassigny, 21079 Dijon, France. ²Service de réanimation polyvalente, CH Victor Dupouy d'Argenteuil, 69 rue du Lieutenant Colonel Prudhon, 95107 Argenteuil, France. ${ }^{3}$ Service de Réanimation Médicale, CHU de SaintAntoine, 184 rue du faubourg Saint-Antoine, 75012 Paris, France. ${ }^{4}$ Service de Réanimation Médicale, Hôpital Emile Muller, 20 Avenue du Docteur René Laennec, 68100 Mulhouse, France. ${ }^{5}$ Service de Néonatologie, CHU de Lyon, 59 Boulevard Pinel, 69500 Bron, France. ${ }^{6}$ Service de Biostatistiques et Informatique Médicale, Département d'Information Médicale, Bocage University Hospital, Boulevard de Lattre de Tassigny, 21079 Dijon, France. ${ }^{7}$ Service de Réanimation polyvalente, CHD les Oudairies, 89925 La Roche sur Yon Cedex 09, France. ${ }^{8}$ Service de Réanimation polyvalente, $\mathrm{CH}$ de Dieppe, Avenue Pasteur, 76202 Dieppe, France.

\section{Authors' contributions}

JPQ was involved in study conception and design, acquisition of data, analysis and interpretation of data and drafting and critical revision of the manuscript. GP was involved in study conception and design and acquisition of data. JLB was involved in study conception and design and acquisition of data. RC was involved in analysis and interpretation of data and acquisition of data. JPR was involved in analysis and interpretation of data, acquisition of data, and drafting and critical revision of the manuscript. $J R$ was involved in acquisition of data and drafting and critical revision of the manuscript. All authors read and approved the final version of the manuscript.

\section{Competing interests}

The authors declare that they have no competing interests.

Received: 8 October 2009 Revised: 8 December 2009 Accepted: 16 March 2010 Published: 16 March 2010

\section{References}

1. Kreymann $K G$, Berger MM, Deutz NE, Hiesmayr M, Jolliet $P$, Kazandjiev $G$, Nitenberg G, Berghe van den G, Wernerman J, Ebner C, Hartl W, Heymann C, Spies C, ESPEN (European Society for Parenteral and Enteral Nutrition): ESPEN Guidelines on Enteral Nutrition: Intensive care. Clin Nutr 2006, 25:210-223.

2. Cerra FB, Benitez MR, Blackburn GL, Irwin RS, Jeejeebhoy K, Katz DP, Pingleton SK, Pomposelli J, Rombeau JL, Shronts E, Wolfe RR, Zaloga GP: Applied nutrition in ICU patients. A consensus statement of the American College of Chest Physicians. Chest 1997, 111:769-778.

3. Thuong M, Leteurtre S: Expert recommendations of the Société de Réanimation de Langue Française: Enternal nutrition in critical care. Réanimation 2003, 12:350-354.

4. Adam S, Batson S: A study of problems associated with the delivery of enteral feed in critically ill patients in five ICUs in the UK. Intensive Care Med 1997, 23:261-266.

5. De Jonghe B, Appere-De-Vechi C, Fournier M, Tran B, Merrer J, Melchior JC, Outin $\mathrm{H}$ : A prospective survey of nutritional support practices in intensive care unit patients: what is prescribed? What is delivered?. Crit Care Med 2001, 29:8-12.

6. Roberts SR, Kennerly DA, Keane D, George C: Nutrition support in the intensive care unit. Adequacy, timeliness, and outcomes. Crit Care Nurse 2003, 23:49-57.

7. Heyland DK, Dhaliwal R, Day A, Jain M, Drover J: Validation of the Canadian clinical practice guidelines for nutrition support in 
mechanically ventilated, critically ill adult patients: results of a prospective observational study. Crit Care Med 2004, 32:2260-2266

8. Mackenzie SL, Zygun DA, Whitmore BL, Doig CJ, Hameed SM: Implementation of a nutrition support protocol increases the proportion of mechanically ventilated patients reaching enteral nutrition targets in the adult intensive care unit. JPEN J Parenter Enteral Nutr 2005, 29:74-80.

9. Koretz RL, Avenell A, Lipman TO, Braunschweig CL, Milne AC: Does enteral nutrition affect clinical outcome? A systematic review of the randomized trials. Am J Gastroenterol 2007, 102:412-429, quiz 468.

10. Stratton RJ, Elia M: Who benefits from nutritional support: what is the evidence?. Eur J Gastroenterol Hepatol 2007, 19:353-358.

11. Le Gall JR, Lemeshow S, Saulnier F: A new Simplified Acute Physiology Score (SAPS II) based on a European/North American multicenter study. JAMA 1993, 270:2957-2963.

12. Binnekade JM, Tepaske R, Bruynzeel P, Mathus-Vliegen EM, de Hann RJ: Daily enteral feeding practice on the ICU: attainment of goals and interfering factors. Crit Care 2005, 9:R218-225.

13. O'Meara D, Mireles-Cabodevila E, Frame F, Hummell AC, Hammel J, Dweik RA, Arroliga AC: Evaluation of delivery of enteral nutrition in critically ill patients receiving mechanical ventilation. Am J Crit Care 2008, 17:53-61.

14. Petros S, Engelmann L: Enteral nutrition delivery and energy expenditure in medical intensive care patients. Clin Nutr 2006, 25:51-59.

15. Harris JA, Benedict FG: Standard basal metabolism constants for physiologists and clinicians: a biometric study of basal metabolism in man. Washington, DC: Carnegie Institution of Washington 1919

16. McClave SA, Sexton LK, Spain DA, Adams JL, Owens NA, Sullins MB, Blandford BS, Snider HL: Enteral tube feeding in the intensive care unit: factors impeding adequate delivery. Crit Care Med 1999, 27:1252-1256.

17. Mentec H, Dupont H, Bocchetti M, Cani P, Ponche F, Bleichner G: Upper digestive intolerance during enteral nutrition in critically ill patients: frequency, risk factors, and complications. Crit Care Med 2001, 29:1955-1961.

18. Jolliet P, Pichard C, Biolo G, Chiolero R, Grimble G, Leverve X, Nitenberg G, Novak I, Planas M, Preiser JC, Roth E, Schols AM, Wernerman J: Enteral nutrition in intensive care patients: a practical approach. Working Group on Nutrition and Metabolism, ESICM. European Society of Intensive Care Medicine. Intensive Care Med 1998, 24:848-859.

19. Desachy A, Clavel M, Vuagnat A, Normand S, Gissot V, Francois B: Initial efficacy and tolerability of early enteral nutrition with immediate or gradual introduction in intubated patients. Intensive Care Med 2008, 34:1054-1059.

20. McClave SA, Lukan JK, Stefater JA, Lowen CC, Looney SW, Matheson PJ, Gleeson K, Spain DA: Poor validity of residual volumes as a marker for risk of aspiration in critically ill patients. Crit Care Med 2005, 33:324-330.

21. Metheny NA, Schallom L, Oliver DA, Clouse RE: Gastric residual volume and aspiration in critically ill patients receiving gastric feedings. Am J Crit Care 2008, 17:512-519, quiz 520.

22. Jain MK, Heyland D, Dhaliwal R, Day AG, Drover J, Keefe L, Gelula M: Dissemination of the Canadian clinical practice guidelines for nutrition support: results of a cluster randomized controlled trial. Crit Care Med 2006, 34:2362-2369.

23. Heyland DK, Dhaliwal R, Drover JW, Gramlich L, Dodek P: Canadian clinical practice guidelines for nutrition support in mechanically ventilated, critically ill adult patients. JPEN J Parenter Enteral Nutr 2003, 27:355-373.

doi:10.1186/cc8915

Cite this article as: Quenot et al:: Bedside adherence to clinical practice guidelines for enteral nutrition in critically ill patients receiving mechanical ventilation: a prospective, multi-centre, observational study. Critical Care 2010 14:R37.

\section{Submit your next manuscript to BioMed Central and take full advantage of:}

- Convenient online submission

- Thorough peer review

- No space constraints or color figure charges

- Immediate publication on acceptance

- Inclusion in PubMed, CAS, Scopus and Google Scholar

- Research which is freely available for redistribution

Submit your manuscript at www.biomedcentral.com/submit 